\title{
Applying TPM on Production Line of Huanwei
}

\author{
Yunkai Zhai ${ }^{1,2,3}$ and Jing Zhang ${ }^{2}$ \\ ${ }^{1}$ Management Engineering School, Zhengzhou University, Zhengzhou, People's Republic of China \\ ${ }^{2}$ Henan Engineering Research Center of Digital Medicine, Zhengzhou, People's Republic of China \\ ${ }^{3}$ The first Affiliate Hospital, Zhengzhou University, Zhengzhou, People's Republic of China \\ Corresponding Email: 1174995417@qq.com
}

\begin{abstract}
As customers increasingly focus on the quality, cost, delivery time of the product, therefore the production line needs to improve production efficiency, improve product quality, which requires the introduction of a management system. The TPM (Total Productive maintenance) as a starting point to maintain the equipment, maintenance activities through full participation in the maintenance, increase productivity, reduce production costs.

The study based on the study and analysis of the TPM theory, based on my practice in the SMT2 production line of Victoria Electronics of Huanwei. Then it studied the promotion and implementation steps of TPM on SMT2 line. And it also introduced the improvement instance of SMT2 line. By contrasting the utilization of equipment on SMT2 line before and after the comprehensive of TPM, the necessity of implementing TPM is derived. For how to apply TPM on the production lines, and how to improve equipment comprehensive utilization, finish the order on time, provide high quality products to our customers, these are the main part of production line equipment management work. This article provides the reference for how to promote TPM on other production lines.
\end{abstract}

\section{Introduction}

\section{1 research background and significance}

Huanwei is an electronics manufacture to provide professional design services and solutions. It mainly for the domestic and international famous brands to provide the design and the material procurement of electronics, computers, phone and other five categories of electronic products.

There exists some problems in the production workshop SMT2 line in Huanwei.The cohesion is unreasonable, and production equipment has problems. So this article is based on SMT2 line, through the data that I collected.The data is what unreasonable about SMT2 line.Through the implying of TPM, we can increase the economic benefit.The application of TPM can raise the production efficiency, and to reduce production costs and to ensure the quality of the products, and to improve the utilization rate of the production workshop equipment.

\section{2 research status}

According to the fire brigade and the fire fighting equipment,and the operator's skill is at low status, Li Kailian, Yang yong [1] thought that carrying out "six big shots"(full equipment repair and maintenance construction) is urgent. Weihong wang, FanTingJiu [2] published the main points of medical equipment maintenance. It based on TPM management deals with the essentials of management of medical equipment maintenance, so as to promote medical equipment maintenance and management, and improve the utilization rate of medical equipment. Fan Yanjiang [3] mainly analyzed the eight generation of Beijing dongfang. He mainly studied the line array factory machinery and equipment management, and product quality, and production efficiency and cost and so on.

Song Guimin [4] pointed out the current pressure of equipment management, and he advocated the participation of the management of machinery and equipment.He also started from the actual situation of enterprises, and guided the operation of equipment field management practices. Elaine Aspinwall, Maged Elgharib [5] determined how to apply TPM in the large and medium-sized manufactures, and analysed why we introduced TPM process, and the results.

Yuan Shan [6] provided the basis to the research of TPM in the aspect of employee. Wang Chunsheng [7] provided scientific basis for the maintenance work. Zhu Haimin [8] imported TPM in the management of Jiangling foundry machinery and equipment. Adnan Hj.Bakri [9] further researched on integrated field of TPM and lean production. 


\section{The theory of TPM}

TPM is total production maintenance.TPM emphasis on participation, such as through full participation, so that it can eliminate waste, and control the whole process, and improve the comprehensive utilization of equipment, and achieve the benefit maximization. TPM is a combination of methods, equipment and operators.Through improving operations, and equipment, we can change the thinking of the employees, and mainly to promote employee's ownership, so that improve the ability of the enterprise to adapt to the external environment.

TPM is an enterprise that improve the system through the improvement of equipment. Advanced machinery and equipment management is the most effective and simple way of increasing efficiency, and reducing the operating costs. TPM has certain coordination effect between each function in manufacturing enterprises. One of the most important is the effect between the two functions of maintain and production. The main goal of TPM is to improve the OEE of equipment. It likes to take measures to reduce or even eliminate the six big losses: the loss of construction, the loss of waste, the machine short outage losses because of machinery and equipment speed down operation, idle equipment, machinery and equipment, adjustment, setting downtime losses, machinery and equipment downtime time loss, etc.

\section{The analysis of the problem of STM2 line}

\subsection{STM2 line}

SMT2 line process is divided into:

Into the moistureproof ark $\rightarrow$ The moistureproof ark $\rightarrow$ Into the station $\rightarrow$ the flap $\rightarrow$ SP2 $\rightarrow$ SPI $\rightarrow$ The patch (iron, connectors $) \rightarrow$ AOI2-F(furnace before test) $\rightarrow$ Reflow soldering $\rightarrow$ Collecting plate $\rightarrow$ AOI2 - B (furnace) $\rightarrow$ SMT2 overall check

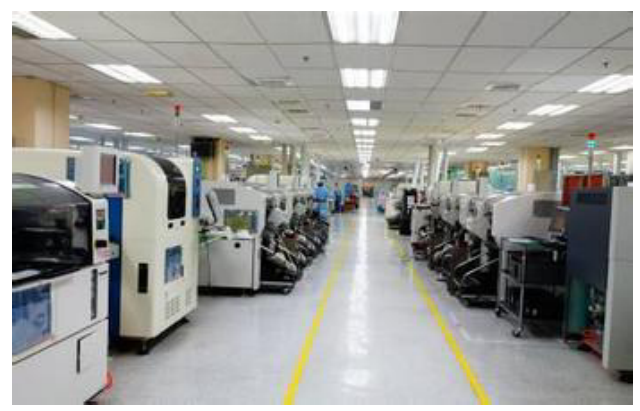

Fig.1. SMT2 line.

\subsection{The problem of STM2 line}

(1) We need to improve equipment management system, and improve the corresponding management mechanism

For SMT2 line, in order to ensure that equipment management work can be arranged on effectively for a long time, we need to establish and improve a series of related equipment management mechanism, and specify the responsibility of the management team and related staff. In apple watches front chip production process, there are many different kinds of chips and operating personnel, machinery and equipment transfers is frequent, and imperfect management mechanism, personnel strength is weak, the phenomenon is common on the production line.

(2) The disconnection between using and maintenance of machinery and equipment

On SMT2 line, even if the staff is assigned to the relatively fixed work, each staff has a corresponding machinery and equipment, but they did not implement machine equipment maintenance work of equipment maintenance personnel and staff. When there is serious problems, equipment maintenance personnel and operators have begun to pass the buck to each other.

(3) The maintenance of machinery and equipment lags, and the waste is serious

On the SMT2 line, daily maintenance measures can not get effective implementation. In the maintenance management of machinery and equipment, the concept of "maintenance" is already entrenched. In such aspects as material, manpower, financial resources, energy, causing unnecessary waste. A lot of equipment maintenance personnel, in order to save time and effort, the materials are useful on equipment repair not recycled, but its subjective to scrap processing.

\subsection{The solution of the existing problem}


(1) Improve the equipment management system

Training employees with TPM at all levels of production line; improving staff quality; making them more clearly about their own work; strengthening the management between leader and employees; ensuring the smooth implementation of equipment management system.

(2) The disconnect between using and maintenance of machinery and equipment

From the two aspects of operator and staff, improving the skill of operators and make them solve the problem of machine timely; and strengthening the sense of ownership of them. Professional maintenance employees should make regular maintenance strategy according to the collection of equipment fault information.

(3) The aintenance of machinery and equipment lags, and the waste is serious

Through the implementation of TPM, all related staff should participate in the machinery and equipment maintenance. When the problems occur, the operator can according to himself technology maintenance first; at the same time, he should inform the professional operators to the scene for further processing.

\subsection{The effect on the SMT2 line}

(1) The OEE calculation before implement TPM

To placement machine on SMT2 line as the research object to show that the utilization rate of machinery and equipment changes after implement TPM on SMT2 line.

Through the observation records, each shift work in $10.5 \mathrm{~h}$, stop $30 \mathrm{~min}$ before the meeting. The production process downtime per class 4 times, and each time the fault processing time for $20 \mathrm{~min}$. More refueling dish 10 times the average class, and each time for $5 \mathrm{~min}$. SMT machine theory for each Carrier chips processing time of $0.25 \mathrm{~min} / \mathrm{Carrier}$, the actual processing cycle $0.5 \mathrm{~min} /$ Carrier. So daily processing chip 550 Carrier, there are 10 Carrier.

On the basis of the above data, before implement TPM SMT2 line, comprehensive utilization calculation to SMT machine are as follows:

Load time $=630 \mathrm{~min}-30 \mathrm{~min}=600 \mathrm{~min}$

Start time $=600 \mathrm{~min}-80 \mathrm{~min}-50 \mathrm{~min}=470 \mathrm{~min}$

Rate of time to start $=470 / 600=78.3 \%$

Rate of speed to start $=0.25 / 0.5=50 \%$

Rate of net working $=550 * 0.5 / 470=58.5 \%$

Rate of working $=50 \% * 58.5 \%=29.2 \%$

Rate of qualified products $=(550-10) / 550=98.2 \%$

So, we can get that:

$\mathrm{OEE}=78.3 \% * 29.2 \% * 98.2 \%=22.5 \%$

(2) The OEE calculation after implement TPM

Through to implement TPM in SMT2 line is for SMT machine, if the fault just reduce 1 time, fault handling time can reduce $2 \mathrm{~min}$, and waste reduce 4 carrier, due to the shorter downtime, chip production increase of 580 carrier, then time statistics are as follows:

In various divisions work $10.5 \mathrm{~h}$, and stop $30 \mathrm{~min}$ before the meeting, the production process downtime per class 3 times, each time the fault processing time for $18 \mathrm{~min}$, more refueling dish 10 times the average class, each time for 5 min, the processing time of a placement machine for each Carrier chip $0.25 \mathrm{~min} /$ Carrier, the actual processing cycle 0.5 min/Carrier, processing chip Carrier 580 a day, six Carrier.

Load time $=630 \mathrm{~min}-30 \mathrm{~min}=600 \mathrm{~min}$

Start time $=600 \mathrm{~min}-54 \mathrm{~min}-50 \mathrm{~min}=496 \mathrm{~min}$

Rate of time to start $=496 / 600=82.7 \%$

Rate of speed to start $=0.25 / 0.5=50 \%$

Rate of net working $=580 * 0.5 / 496=58.5 \%$

Rate of working $=50 \% * 58.5 \%=29.2 \%$

Rate of qualified products $=(580-6) \quad / 580=99.0 \%$

So,we can get that:

$\mathrm{OEE}=82.7 \% * 29.2 \% * 99.0 \%=23.9 \%$

(3) The OEE contrast before and after implementing TPM

The OEE for SMT2 line placement machine calculated above. Before and after implementing TPM, placement machine OEE improved about:

$23.9 \%-22.5 \%=1.4 \%$

Although the comprehensive utilization ratio of SMT machine only increased by $1.4 \%$, for SMT2 line, has been greatly improved.

\section{Summary}


The purpose of this paper is the study of TPM on SMT2 line on the popularization and application. Application TPM on SMT2 line, not only can improve the comprehensive utilization of equipment, and improves the staff maintenance machinery and equipment of self-consciousness, also can improve the morale of the team members, and to improve the factory environment, and decrease the wasting of human resources. TPM is not just a policy, it also includes the production line on the organization structure change, full participation. Predictable, after the TPM really applied SMT2 line will have a lot of improvement.

\section{References}

1. Li Kailian, Yang yong. TPM full-time fire team in the enterprise management application. Journal of fire monthly, 2002, 28 (7) : 21-36.

2. Wang Wei-hong, FanTingJiu. Based on the TPM management of medical equipment maintenance points. China medical equipment, 2008, 11:88-89.

3. Fan Yanjiang. TPM in the application of liquid crystal display manufacturing boe research. Beijing: university of Chinese academy of sciences, 2014.

4. Song Guimin. Total productive maintenance equipment management new method. Journal of chemical engineering and equipment, 2012, 10 (5) : 46-58.

5. Elaine Aspinwall, Maged Elgharib. TPM implementation in large and medium size orginazations. Journal of Manufacturing Technology Management, 2013 (05) : 56-77.

6. Yuan Shan, photo, Wang Bulin. Total productive maintenance mode using the thinking of the unity of operating check. Science and technology of meishan, 2014 01:59-61.

7. Wang Chunsheng. Systemic power plant equipment maintenance mode and its preliminary application. North China electric power university, 2001.

8. Zhu Haimin. The application of TPM in jiangling foundry research. Nanjing: nanjing university of science and technology, 2006:41-65.

9. Adnan Hj.Bakri,Abdul Rahman Abdul Rahim,Noordin Mohd,Yusof Ramlli Ahmad. Boosting Lean Production via TPM.Procedia-Social and Behavioral Sciences, 2012, 21(05): 20-37. 